\title{
Tidal stabilization of rigidly rotating, fully relativistic neutron stars
}

\author{
Kip S. Thorne \\ Theoretical Astrophysics, California Institute of Technology, Pasadena, California 91125
}

(Received 17 June 1997; published 23 November 1998)

\begin{abstract}
It is shown analytically that an external tidal gravitational field increases the secular stability of a fully general relativistic, rigidly rotating neutron star that is near marginal stability, protecting it against gravitational collapse. This stabilization is shown to result from the simple fact that the energy $\delta M(\mathcal{Q}, R)$ required to raise a tide on such a star, divided by the square of the tide's quadrupole moment $\mathcal{Q}$, is a decreasing function of the star's radius $R,(d / d R)\left[\delta M(\mathcal{Q}, R) / \mathcal{Q}^{2}\right]<0$ (where, as $R$ changes, the star's structure is changed in accordance with the star's fundamental mode of radial oscillation). If $(d / d R)\left[\delta M(\mathcal{Q}, R) / \mathcal{Q}^{2}\right]$ were positive, the tidal coupling would destabilize the star. As an application, a rigidly rotating, marginally secularly stable neutron star in an inspiraling binary system will be protected against secular collapse, and against dynamical collapse, by tidal interaction with its companion. The "local-asymptotic-rest-frame" tools used in the analysis are somewhat unusual and may be powerful in other studies of neutron stars and black holes interacting with an external environment. As a by-product of the analysis, in an appendix the influence of tidal interactions on mass-energy conservation is elucidated. [S0556-2821(98)05222-9]
\end{abstract}

PACS number(s): 04.40.Dg, 04.30.Db, 97.60.Jd, 97.80.-d

\section{INTRODUCTION AND SUMMARY}

Wilson, Mathews, and Maronetti [1] have carried out fully relativistic numerical simulations of the radiationreaction-induced inspiral of a binary neutron star system. To make their computations tractable, they employed several approximations of ill-understood accuracy. The stars in their simulations were identical and were near the maximum allowed mass for an isolated neutron star. Correspondingly, when the stars were far apart in their orbit, each was stable against gravitational collapse. Surprisingly, as the stars spiraled inward, the simulations indicated that their gravitational interaction destabilized them, triggering them to collapse before their inspiral ended. The magnitude of the destabilization and mathematical arguments to explain it [1] suggest that it should show up in the first post-Newtonian approximation to general relativity.

Several researchers have argued that this surprising destabilization is wrong: Lai [2] has shown that tidal interactions between two nearly Newtonian stars will tend to stabilize them against gravitational collapse, not destabilize them. Lai's stabilization effect is formally of Newtonian origin, but because of the compactness of neutron stars, its magnitude is of much higher post-Newtonian order. Wiseman [3] has elucidated Lai's conclusion by showing that at first postNewtonian magnitude, the stars' gravitational interactions do not alter their individual central densities, and Brady and Hughes [4] have shown the same at first order in the mass ratio $M_{2} / M_{1}$ when the two stars are fully relativistic and one is much less massive than the other, $M_{2} \ll M_{1}$. Baumgarte et al. [5] have carried out numerical simulations of the fully relativistic equilibrium states of a binary neutron star system in synchronous, circular orbit-simulations analogous to those of Wilson, Mathews, and Maronetti. Not only do these simulations show no sign of interaction-induced collapse; when combined with "turning-point" criteria for secular stability, they actually reveal a stabilization of the stars.

On the basis of these analyses, it seems likely that the destabilization seen by Wilson, Mathews, and Maronetti does not occur in reality, but instead is an artifact of pooraccuracy approximations or is due to some error in their computations.

In this paper we present another analysis that reveals stabilization, not destabilization. Our justification for yet another paper on this subject is twofold: First, our analysis has broader validity than previous ones-it is fully relativistic, not post-Newtonian, and unlike the two previous fully relativistic analyses of stability [1,5], it is fully analytic and not based on numerical simulations; it permits the stars to rotate with arbitrary angular velocity (though with spins aligned with the orbital angular momentum), and it allows an arbitrary mass ratio. Second, our analysis employs an unusual approach, which may be useful for other problems in fully relativistic binary evolution: it is formulated in the local asymptotic rest frame of one of the two stars and employs energy and angular momentum arguments that relate to that star alone and not to the binary system as a whole. Although this approach is unusual within general relativity, it is well known in Newtonian and post-Newtonian theory. It, in fact, is a relativistic generalization of Lai's [2] post-Newtonian proof of stabilization.

This paper is organized as follows: In Sec. II we treat an idealized problem that illustrates our method: the stabilization of a non-spinning neutron star placed in a non-rotating external tidal gravitational field. Then in Sec. III we generalize to a spinning star and rotating tidal field, and as an application we deduce the secular stabilization of a spinning neutron star in an inspiraling binary. In Sec. IV we argue from this secular stabilization result that, if an inspiraling binary's neutron stars are secularly stable at large orbital radii, then they cannot be dynamically destabilized during the inspiral, and we make some concluding remarks. In an appendix we elucidate the influence of tidal interactions on energy conservation, in the Newtonian limit. The relativistic version of this issue is a central aspect of the proof of stability given in the body of the paper. 


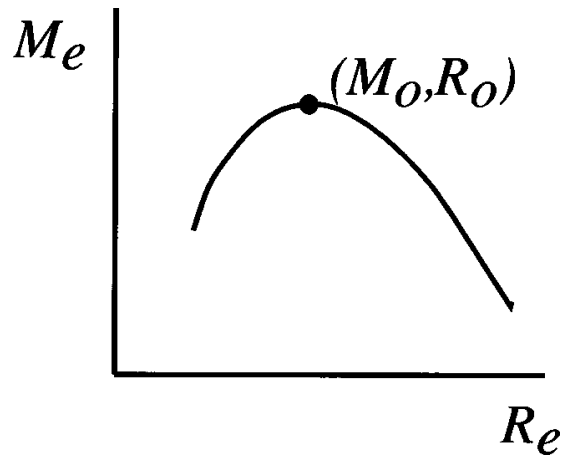

FIG. 1. The mass-radius curve for static, spherical equilibrium configurations (neutron stars) with some equation of state $P(\rho)$.

Throughout we use geometrized units in which the speed of light and Newton's gravitation constant are unity.

\section{STATIC STAR AND STATIC TIDAL FIELD}

\section{A. Momentarily static, spherical star}

Consider a family of nonrotating, spherical neutron stars characterized by a one-parameter equation of state $P$ $=P(\rho)$, where $P$ is pressure and $\rho$ is density of total massenergy. We shall refer to these stars as equilibrium configurations and shall denote their masses (as measured by the Keplerian orbits of distant planets) by $M_{e}$, their total number of baryons by $N_{e}$, and their radii (defined as circumference $/ 2 \pi)$ by $R_{e}$. The mass-radius relation $M_{e}\left(R_{e}\right)$ of these equilibrium configurations has the qualitative form shown in Fig. 1], and $N_{e}\left(R_{e}\right)$ has a similar shape; cf. Refs. $[6,7]$.

As is well known, the equilibrium configuration of maximum mass (solid circle in Fig. 1, critical configuration, mass $M_{o}$ and radius $R_{o}$ ) is secularly marginally stable: It possesses a zero-frequency mode of expansion (or contraction) that takes it horizontally in the figure to another equilibrium configuration with the same mass. Equilibria on the largerradius side of the critical configuration are secularly stable; those on the lower-radius side are secularly unstable [6,7].

By secular is meant a mode of stellar deformation which is slow enough for pycnonuclear reactions (pressure-induced nuclear reactions) to keep its matter always at the end point of nuclear evolution; so the pressure and density changes experienced by the stellar matter follow the same equation of state $P(\rho)$ as characterizes the equilibrium configuration itself. The slowest of the pycnonuclear reactions are "modified URCA reactions"' (essentially $\beta$ and inverse- $\beta$ decays), driven as the star deforms by the rising or falling Fermi energies of the star's electrons, protons, and neutrons; they can require time scales of minutes or longer to equilibrate near and below nuclear densities, and so in principle the stellar deformations can be secular only on time scales longer than this. (In practice, these slow reactions have only a weak effect on the equation of state near and above nuclear densities; so their slowness is often ignored for near critical neutron stars.)

Faster (dynamical) motions, in which some of the pycnonuclear reactions do not go to completion, will be character- ized by a stiffer equation of state (higher adiabatic index) than secular motions and thus will be more stable. Correspondingly, all equilibria on the large- $R_{e}$ branch, being secularly stable, must also be dynamically stable, as must be the critical configuration itself. For an ancient, further discussion of these issues but in a different language, see Ref. [8]

In the next subsection we shall study the influence of an external tidal field on the secular stability of configurations that are nearly critical (nearly at the maximum of the massradius curve). As an aid in that study, it will be useful to consider momentarily static, spherical stars that are deformed slightly away from equilibrium. For a star containing $N$ baryons (with $N$ very nearly equal to $N_{o}$ ), we obtain such a non-equilibrium configuration as follows: Begin with the equilibrium configuration that has $N_{e}=N$ and radius $R_{e}\left(N_{e}\right)$. Expand it or contract it slightly to the desired new radius $R$. In this deformation, displace each fluid element by an amount proportional to the equilibrium configuration's fundamental-mode eigenfunction $\vec{\xi}(\vec{x})$ of secular vibration, and then remove all kinetic energy of deformation. The resulting deformed configuration will have a mass $M(N, R)$ that differs slightly from the equilibrium mass $M_{e}\left(N_{e}=N\right)$.

In practice, the mass of the deformed star can be computed using not the star's true eigenfunction, but rather the eigenfunction $\vec{\xi}_{o}(\vec{x})$ of the zero-frequency mode of the critical configuration. This is because the mass is an extremum with respect to deformations of the star [6]; the two eigenfunctions $\vec{\xi}$ and $\vec{\xi}_{o}$ differ by fractional amounts of order $n$ $\equiv\left(N-N_{o}\right) / N_{o}$, and so the masses of the configurations obtained by deformations to radius $R$ via the $\vec{\xi}$ motion and the $\vec{\xi}_{o}$ motion will differ by a fractional amount of order $n^{2}$-which is never of interest in this paper.

The configurations obtained by the above construction are characterized by two parameters $(N, R)$, and their masses are functions of these parameters, $M(N, R)$.

For ease of analysis, we shall now convert to dimensionless variables:

$$
m \equiv \frac{M-M_{o}}{M_{o}}, \quad n \equiv \frac{N-N_{o}}{N_{o}}, \quad r \equiv \frac{R-R_{o}}{R_{o}} .
$$

These variables characterize a configuration's fractional deviations from the critical configuration.

Figure 2 shows the dimensionless mass-radius relation $m(n, r)$ for configurations with fixed baryon number $n$ (dashed curves), along with the equilibrium configurations (solid curve). Because the equilibria on the positive- $r$ branch are stable against secular deformations (with eigenfunction $\vec{\xi}$ ), they lie at minima of the dashed curves; because those on the negative- $r$ branch are unstable, they lie at maxima of the dashed curves.

These dashed curves, when expressed as a power series, have the following form:

$$
m=a r^{3}+\left(b_{0}+b_{1} r\right) n
$$

where higher-order terms are of no importance in this paper, and where $a, b_{0}$, and $b_{1}$ are all positive. This form is dic- 


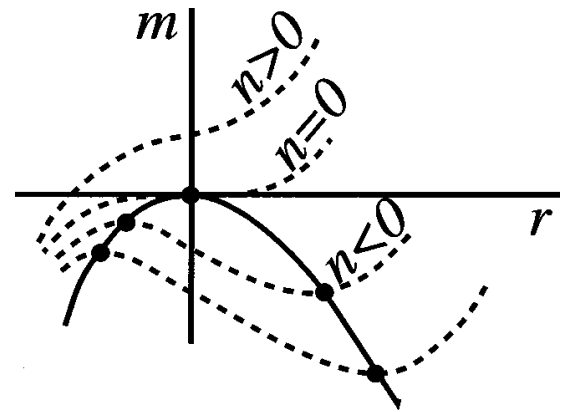

FIG. 2. Solid curve: the dimensionless mass-radius relation for the equilibrium configurations of Fig. 1. Dashed curves: the dimensionless mass-radius relations $m(n, r)$ for configurations of fixed baryon number $n$ that are obtained from an equilibrium configuration via deformation along the fundamental eigenfunction of radial secular motion.

tated by the demand that for $n=0$ the dashed curve be flat at $r=0$, that for $n<0$ the positive- $r$ branch have a minimum (stable equilibrium) and the negative- $r$ branch have a maximum (unstable), and that for $n>0$ there be no equilibria at all (no extrema of the dashed curves).

The equilibrium configurations are located at the extrema of these mass-radius curves, i.e. at locations where $(\partial m / \partial r)_{n}=0$, which yields the following equilibrium relations:

$$
\begin{aligned}
& n_{e}=\frac{-3 a}{b_{1}} r_{e}^{2}, \quad m_{e}=\frac{-3 a b_{0}}{b_{1}} r_{e}^{2}-2 a r_{e}^{3}, \\
& m_{e}=b_{0} n_{e} \mp 2 \frac{\left(b_{1} / 3\right)^{3 / 2}}{a^{1 / 2}}\left(-n_{e}\right)^{3 / 2},
\end{aligned}
$$

where the upper sign is for the right branch (stable stars, lower mass at fixed $n_{e}$ ) and the lower sign, for the left branch (unstable stars, higher mass at fixed $n_{e}$ ).

The coefficients $a, b_{0}$, and $b_{1}$ are determined as follows in terms of the equilibrium configurations: As is well known —cf. Eq. (28) of Ref. [6] - the mass-energy required to create one baryon and inject it into an arbitrary location in an equilibrium configuration, in local thermodynamic equilibrium with the matter there, is $d M_{e} / d N_{e}$ $=\mu_{B} \sqrt{1-2 M_{e} / R_{e}}$, where $\mu_{B}$ is the rest mass of one baryon at the star's surface $\left(1 / 56\right.$ the mass of an ${ }^{56} \mathrm{Fe}$ nucleus if the star's matter has been "catalyzed to the end point of thermonuclear evolution" [6]). Evaluating this "injection energy" for the critical configuration, switching to dimensionless variables, and comparing with $\left(d m_{e} / d n_{e}\right)_{o}=b_{0}$ [Eq. (3)], we see that

$$
b_{0}=\left(\mu_{B} N_{o} / M_{o}\right) \sqrt{1-2 M_{o} / R_{o}} \sim 0.8 .
$$

Performing the same calculation slightly away from the critical configuration, we obtain

$$
b_{1}=\frac{\mu_{B} N_{o} / R_{o}}{\sqrt{1-2 M_{o} / R_{o}}} \sim 0.6
$$

The remaining coefficient, $a$, is determined by the curvature $\left(d^{2} M_{e} / d R_{e}^{2}\right)_{o}$ of the equilibrium mass-radius relation at its critical point [cf. Eq. (3)]:

$$
a=\frac{R_{o}}{6\left(1-2 M_{o} / R_{o}\right)}\left(\frac{-d^{2} M_{e}}{d R_{e}^{2}}\right)_{o} \sim 1 .
$$

In Eqs. (4) and later equations in this paper, the numerical values have been inferred, with uncertainties typically no worse than a factor of 2 , from the equilibrium configurations for plausible equations of state [7].

\section{B. Static, tidally deformed star}

We now place a near-critical neutron star in a static, external tidal gravitational field, which we characterize by the space-time-space-time components of its Riemann tensor, $R_{j 0 k 0} \equiv \mathcal{E}_{j k}$ [9]. This tidal field will deform the star, i.e. will gravitationally "polarize" it, giving it a gravitational mass quadrupole moment $\mathcal{I}_{j k}$. The tidal field $\mathcal{E}_{j k}$, quadrupole moment $\mathcal{I}_{j k}$, and total stellar mass $\mathcal{M}$ (including the deformation energy and the energy of interaction between the deformation and the tidal field) all show up as coefficients in a power series expansion of the spacetime metric in the star's local asymptotic rest frame [10]. ${ }^{1}$ For example, in harmonic (deDonder) coordinates that are attached to the star's center of mass, the time-time metric component has the form $[9,10,12]$

$$
\begin{aligned}
g_{00}= & -1+2 \frac{\mathcal{M}}{r}-2 \frac{\mathcal{M}^{2}}{r^{2}}+2 \frac{\mathcal{M}^{3}}{r^{3}}+3 \frac{\mathcal{I}_{j k} n^{j} n^{k}}{r^{3}}+\ldots \\
& -\mathcal{E}_{j k} n^{j} n^{k} r^{2}+\ldots,
\end{aligned}
$$

where the first ellipsis denotes higher-order terms in $1 / r$ and the second ellipsis denotes higher-order terms in $r$. Here, and only here, $r$ is the coordinate radius computed as though the spatial coordinates $x^{j}$ were precisely Cartesian [elsewhere in this paper $r \equiv\left(R-R_{o}\right) / R_{o}$ is the star's dimensionless radius], and $n^{j} \equiv x^{j} / r$ is the "unit radial vector." The local asymptotic rest frame, where the expansion (5) is valid, is the region from the neutron star's surface out to a distance where the external tidal field can no longer be regarded as uniform.

For simplicity, and in accordance with the case of a tidal field produced by a distant binary companion, we shall assume that the tidal field is axisymmetric, and we shall choose our (nearly Cartesian) harmonic coordinates so that its symmetry axis is along the $x^{3}$ direction. Then the induced quad-

\footnotetext{
${ }^{1}$ Reference [10] is the principal conceptual and mathematical foundation for this paper's analysis. The physical concepts that underlie Ref. [10] and this paper, including the validity of the equivalence principle for "extended" self-gravitating bodies such as neutron stars, date back to Wheeler's discussion of equations of motion in Sec. 20.6 of Misner, Thorne, and Wheeler (MTW) [11] and to references therein.
} 
rupole moment also possesses this symmetry, and the components of the tidal field and quadrupole moment take the form

$$
\mathcal{E}_{x x}=\mathcal{E}_{y y}=-\frac{1}{2} \mathcal{E}_{z z} \equiv \mathcal{E}, \quad \mathcal{I}_{x x}=\mathcal{I}_{y y}=-\frac{1}{2} \mathcal{I}_{z z} \equiv-\mathcal{Q} .
$$

Here the signs are chosen such that $\mathcal{E}$ and $\mathcal{Q}$ are both positive.

In the next few paragraphs, culminating with Eq. (15), we shall compute the magnitude $\mathcal{Q}$ of the quadrupole moment that is induced by a given tidal field $\mathcal{E}$. We do so by the following thought experiment: Begin with a near critical, spherical configuration of mass $M$, baryon number $N$, and radius $R$, and turn on the tidal field $\mathcal{E}$ without letting the star deform. Then allow the star to deform of its own accord, in response to the fixed tidal field $\mathcal{E}$. The deformation will develop on the time scale of the star's $f$-mode quadrupolar oscillations, ${ }^{2} \sim 0.3 \mathrm{msec}$, which is far faster than the star's radial motions; thus, its angle-averaged radius $R$ will remain essentially unchanged during the deformation. As the deformation proceeds, i.e. as $\mathcal{Q}$ grows, the tidal field does work $W(\mathcal{Q}, \mathcal{E})$ on the star; i.e., it increases the star's total massenergy (excluding quadrupole-tidal interaction energy) by that amount. Some portion $\delta M(\mathcal{Q}, R)$ of the work $W$ goes into producing the stellar deformation (pushing mass up in the polar regions and down in the equatorial regions); the rest goes into the kinetic energy of quadrupolar vibrations. Suppose that we extract the kinetic energy; then the star will settle down into the deformed configuration that has released the most kinetic energy, i.e. the configuration that minimizes the potential energy function

$$
V(N, R, \mathcal{Q}, \mathcal{E}) \equiv M(N, R)+\delta M(\mathcal{Q}, R)-W(\mathcal{Q}, \mathcal{E}) .
$$

The quadrupolar deformation energy $\delta M(\mathcal{Q}, R)$ can be deduced in cgs units by dimensional arguments. It obviously must be quadratic in $\mathcal{Q}$, proportional to Newton's gravitation constant $G$ and independent of the speed of light $c$, which means it must have the form $\delta M=\beta G \mathcal{Q}^{2} / R^{5}$ where $R$ is the configuration's radius (the only length scale available other than those, of order $R$, that characterize the star's internal structure). Here $\beta$ is a dimensionless coefficient of order unity that depends on dimensionless aspects of the star's internal structure. In geometrized units $(G=c=1)$, this mass-energy of deformation is

$$
\delta M(\mathcal{Q}, R)=\frac{\beta}{R^{5}} \mathcal{Q}^{2}
$$

This is the same expression as one obtains in Newtonian theory. It can be understood, in Newtonian language, as the gravitational energy $\sim(\Delta M)^{2} / R$ of an excess mass $\Delta M$ $\sim \mathcal{Q} / R^{2}$ moved into the star's polar region a little higher than

\footnotetext{
${ }^{2}$ The tidal field, having no radial nodes in its tidal force pattern, will primarily excite the node-free $f$-mode; it will couple far more weakly to the lower-frequency, longer-time-scale $g$-modes [13-15].
}

radius $R$, and a corresponding mass deficit $-\Delta M$ in the star's equatorial region a little lower than radius $R$. As an example, for a uniform-density Newtonian star [16], $\beta=3$.

The work $W(\mathcal{Q}, \mathcal{E})$, which the fixed tidal field $\mathcal{E}$ does on the star as its quadrupole moment grows from zero to the value $\mathcal{Q}$, can be deduced by examining the flow of energy through the star's local asymptotic rest frame. That energy flow can be described by the Landau-Lifshitz pseudotensor [17] for the metric (5) with $\mathcal{E}$ fixed and $\mathcal{Q}$ time varying. By integrating the pseudotensor over a sphere $\mathcal{S}$ in the star's local asymptotic rest frame, we obtain the rate of change of the star's total mass-energy [the quantity $\mathcal{M}$ appearing in the metric (5)]:

$$
\frac{d \mathcal{M}}{d t}=-\int_{\mathcal{S}}(-g) t_{L L}^{j 0} n_{j} d^{2} A
$$

cf. Secs. 20.2 and 20.3 of Ref. [11]. This $d \mathcal{M} / d t$ consists of two parts: the rate $(d / d t) W(\mathcal{Q}, \mathcal{E})$ that work is done by the external field on the star and the rate of change $d E_{\text {int }} / d t$ of the interaction energy of the external field and the stellar deformation:

$$
\frac{d \mathcal{M}}{d t}=\frac{d W(\mathcal{Q}, \mathcal{E})}{d t}+\frac{d E_{\mathrm{int}}}{d t}
$$

This split of $d \mathcal{M} / d t$ into two parts is elucidated in the Appendix and will be analyzed at greater length in a subsequent paper [18]. The integral (9) has been evaluated by Zhang [19] using techniques described in Ref. [10] and foundations laid in [20]. The result, in a general situation where both $\mathcal{E}$ and $\mathcal{Q}$ may be changing, is ${ }^{3}$

$$
\begin{aligned}
\frac{d \mathcal{M}}{d t} & =\frac{d W(\mathcal{Q}, \mathcal{E})}{d t}+\frac{d E_{\mathrm{int}}}{d t} \\
& =-\frac{1}{2} \mathcal{E}_{i j} \frac{d}{d t} \mathcal{I}^{i j}-\frac{1}{10} \frac{d}{d t}\left(\mathcal{E}_{i j} \mathcal{I}^{i j}\right) \\
& =3 \mathcal{E} \frac{d \mathcal{Q}}{d t}+\frac{3}{5} \frac{d(\mathcal{E} \mathcal{Q})}{d t} .
\end{aligned}
$$

The following argument tells us how much of this massenergy change goes into work and how much into interaction energy: (i) The interaction energy $E_{\text {int }}$ can depend only on the instantaneous stellar deformation and tidal field; so $d E_{\text {int }} / d t$ must always be a perfect differential. By contrast, the rate $d W / d t$ that work is done need not be a perfect differential. (ii) In a physical situation (not ours) where the tidal field is changing while the stellar configuration is constant, to first-order in the tidal perturbation no work is done on the star, $d W / d t=0$. These two facts are sufficient to imply that

\footnotetext{
${ }^{3}$ Zhang does not give explicitly the numerical coefficient $-1 / 10$, since the perfect time derivative term is not of interest for his problem; it can be derived by filling in intermediate steps in Zhang's calculation.
} 


$$
\begin{aligned}
& \frac{d W(\mathcal{Q}, \mathcal{E})}{d t}=-\frac{1}{2} \mathcal{E}_{i j} \frac{d}{d t} \mathcal{I}^{i j}=3 \mathcal{E} \frac{d \mathcal{Q}}{d t}, \\
& \frac{d E_{\mathrm{int}}}{d t}=-\frac{1}{10} \frac{d\left(\mathcal{E}_{i j} \mathcal{I}^{i j}\right)}{d t}=\frac{3}{5} \frac{d(\mathcal{E} \mathcal{Q})}{d t} .
\end{aligned}
$$

In our thought experiment, where the tidal field is fixed at $\mathcal{E}$ and the quadrupole moment grows from zero to $\mathcal{Q}$, the total work done on the star is

$$
W(\mathcal{Q}, \mathcal{E})=-\frac{1}{2} \mathcal{I}_{i j} \mathcal{E}^{i j}=3 \mathcal{Q} \mathcal{E}
$$

By inserting expressions (14) and (8) into Eq. (7) and minimizing the resulting potential energy $V(N, R, \mathcal{Q}, \mathcal{E})$ with respect to $\mathcal{Q}$ at fixed $N, R, \mathcal{E}$, we deduce the equilibrium value of the quadrupole moment:

$$
\mathcal{Q}=\frac{3 R^{5}}{2 \beta} \mathcal{E}
$$

Correspondingly, the potential energy of the quadrupolar equilibrated configuration is

$$
V(N, R, \mathcal{E})=M(N, R)-\frac{9 R^{5}}{4 \beta} \mathcal{E}^{2} .
$$

Because the quadrupolar contribution $-\left(9 R^{5} / 4 \beta\right) \mathcal{E}^{2}$ to this potential energy depends on the star's radius, the tidal coupling influences the star's radial, secular motions. To deduce that influence, we convert the potential energy to dimensionless units,

$$
v(n, r, \varepsilon) \equiv \frac{V(N, R, \mathcal{E})-M_{o}}{M_{o}}, \quad \varepsilon \equiv \frac{\mathcal{E}}{M_{o} / R_{o}^{3}},
$$

and combine with Eqs. (16) and (2) to obtain

$$
v(n, r, \varepsilon)=a r^{3}+\left(b_{0}+b_{1} r\right) n-\left(c_{0}+c_{1} r\right) \varepsilon^{2} .
$$

Here $\varepsilon$ is the external tidal field measured in units of the tidal field produced by the critical configuration near its own surface, and

$$
\begin{aligned}
& c_{0}=\frac{1}{M_{o}}\left(\frac{M_{o}}{R_{o}^{3}}\right)^{2} \frac{9 R_{o}^{5}}{4 \beta}=\frac{9 M_{o}}{4 \beta R_{o}} \sim 0.3, \\
& c_{1}=\frac{R_{o}}{M_{o}}\left(\frac{M_{o}}{R_{o}^{3}}\right)^{2}\left[\frac{d}{d R}\left(\frac{9 R^{5}}{4 \beta}\right)\right]_{o} \simeq 5 c_{0} \sim 1.5,
\end{aligned}
$$

where the factor of 5 comes from differentiating $R^{5}$. The dependence of $\beta$ on the star's internal structure may give rise to a slight variation of $\beta$ with $R$, which may slightly change the relationship $c_{1}=5 c_{0}$, hence the approximate equality in Eq. (19).

As the near critical star changes its radius $R$ slowly and secularly at fixed tidal field $\mathcal{E}$, its quadrupole moment continually equilibrates, with an accompanying tidal feeding of energy $W$ into and out of the star. These radial motions conserve the star's relativistic kinetic energy plus its potential energy $V(N, R, \mathcal{E})$ [which includes a correction for the flow of $W$; cf. Eq. (7)]. Correspondingly, the star's equilibria are the extrema of $V(N, R, \mathcal{E})$ - or equivalently of its dimensionless version $v(n, r, \varepsilon)$ - and these equilibria are stable if they minimize $v$ and unstable if they maximize it.

By differentiating Eq. (18) with respect to $r$ at fixed $n$ and $\varepsilon$, we obtain, for the equilibrium configurations,

$$
\begin{aligned}
r_{e} & = \pm \sqrt{(1 / 3 a)\left(-b_{1} n_{e}+c_{1} \varepsilon^{2}\right)} \\
& \sim \pm \frac{0.7 \mathcal{E}}{M_{o} / R_{o}^{3}} \text { near criticality, where } n_{e} \ll \frac{c_{1}}{b_{1}} \varepsilon^{2} .
\end{aligned}
$$

The configurations on the + branch $\left(R>R_{o}\right)$ are stable, and those on the - branch $\left(R<R_{o}\right)$ are unstable.

Notice that, in response to the tidal field, each stable equilibrium configuration increases its radius $\left(r_{e}>0\right)$, and correspondingly, since the fundamental radial-mode eigenfunction $\vec{\xi}(\vec{x})$ that describes the radial shape of its spherical deformation has no nodes, its central density goes down. Not surprisingly, this leads to a secular stabilization of the star: whereas before the tidal field was turned on the maximum number of baryons that the star could support without collapsing was $N_{o}$, afterward the maximum baryon number has increased by a fractional amount

$$
n_{e \max }=\frac{c_{1}}{b_{1}} \varepsilon^{2} \sim 2.5\left(\frac{\mathcal{E}}{M_{o} / R_{o}^{3}}\right)^{2} .
$$

The tidally induced increase of equilibrium radius (20) and increase of maximum baryon number (21) are the same in sign and order of magnitude as Lai has deduced previously using post-Newtonian arguments [2].

The inability of fully relativistic gravity to produce a secular instability can be traced to the robustly positive sign of the coefficient $c_{1}$, which in turn follows from the fact that the star's deformation energy at fixed quadrupole moment, $\delta M(\mathcal{Q}, R) / \mathcal{Q}^{2}=\beta / R^{5}$, is a decreasing function of radius. It is very hard to imagine any neutron star for which this would fail to be true.

\section{ROTATING STAR AND ROTATING TIDAL FIELD}

We now turn attention to a rigidly rotating neutron star interacting with a rotating external tidal field, such as those which occur in binary neutron star systems. In our analysis, the rotation is with respect to the star's local asymptotic rest frame, which itself will generally rotate with respect to "infinity" due to dragging of inertial frames by the binary's total angular momentum.

For simplicity, we shall require that the star's spin axis and the tidal field's rotation axis coincide, as will be the case in a binary if the two stars' spin axes are perpendicular to the orbital plane. This requirement protects the tidal field and the spin axis from processing.

The tidal stabilization analysis of Sec. II can be generalized to such a rotating system with little change. We shall 
sketch that generalization, beginning in Sec. III A with the secular stability of the rotating star in the absence of the tidal field, followed in Sec. III B with the star-tide interaction and tidal stabilization.

\section{A. Rotating star without a tidal field}

Consider a family of rigidly rotating neutron stars that are characterized by the equation of state $P(\rho)$ and that all have the same spin angular momentum $J$ as measured by frame dragging in their local asymptotic rest frames. For such stars, because $J$ is fixed once and for all, the equilibrium configurations form a one-parameter family analogous to that for static stars. We shall characterize those equilibria by their masses $M_{e}$ (as measured by Keplerian orbits in their local asymptotic rest frames), their number of baryons $N_{e}$, and their equatorial radii $R_{e}$ (defined as their equatorial circumferences $/ 2 \pi$ ).

These equilibria will have a mass-radius relation $M_{e}\left(R_{e}\right)$ with the same general shape as that for nonrotating stars (Fig. 1), but with a critical mass $M_{o}$ and critical radius $R_{o}$ that are larger due to centrifugal stabilization and centrifugal forces; cf., e.g., Figs. 1 and 2 of Ref. [21].

As for static stars, so also for these spinning stars, the critical configuration (configuration of maximum mass) is marginally stable to a secular expansion or contraction that takes it horizontally in Fig. 1 to another equilibrium configuration with the same mass $M_{o}$ and angular momentum $J$; the equilibrium configurations on the larger-radius side of critical are secularly stable, while those on the smaller-radius side are secularly unstable.

In these rotating stars, the motions of interest are secular in two senses: (i) As for static stars, the motions must be slow enough for pycnonuclear reactions to go to completion, so that each element of expanding and contracting stellar material follows the same equation of state $P(\rho)$ as characterizes the equilibrium stellar structure. (ii) The motions must also be slow enough for viscosity to produce enough coupling between adjacent mass elements to keep the star rigidly rotating. This second condition is especially severe; so in practice the secular stabilization that we shall prove is of interest primarily because of its implications for dynamical stability (Sec. IV).

As for static stars, we construct from our one-parameter rigidly rotating family of equilibria a two-parameter family of configurations that are expanded and contracted away from equilibrium. Our construction procedure is precisely the same as in the static case, with the slow, fundamental secular mode of deformation governing the expansions and contractions. The resulting deformed configurations will obviously have mass-radius curves, $M(N, R)$ at fixed $N$ (and forever fixed $J$ ), with the same general shapes as in the static case: the dashed curves of Fig. 2. Moreover, when expressed in dimensionless variables $(1)$, the $m(n, r)$ relation near the critical point for our rotating family will have the same mathematical form, $m=a r^{3}+\left(b_{0}+b_{1} r\right) n$, as for the static case. The coefficients $a, b_{0}$, and $b_{1}$ will be affected by the rotation and thus will not take on their static forms (4), but in order to produce the equilibria's known stability properties, they will still all be positive.

\section{B. Tidally deformed, rotating star}

We now place each of our rigidly rotating, near-critical stars-all with the same spin angular momentum $J$-in a rotating, external tidal gravitational field. We assume that the star and the tidal field rotate about the same axis as seen in the star's local asymptotic rest frame; the star's rotational angular velocity is $\omega \leq 2 \pi \times 1000 / \mathrm{s}$, and if the rotating field is produced by a binary companion, its angular velocity is that of the orbital motion, $\Omega \lesssim 2 \pi \times 600 / \mathrm{s}$ [22]. In this case, the tidal field is exceedingly unlikely to excite the star's normal modes significantly. This is because (i) significant excitation requires resonant coupling; (ii) the circularly polarized $f$ - and $p$-modes have angular velocities of pattern rotation $\sigma \gtrsim 2 \pi \times 1500 / \mathrm{s}$ (unless the star is rotating close to centrifugal breakup), which is too large to resonate with the driving force except under the most extreme of circumstances; and (iii) the low-frequency $g$-modes, which can resonate, have only very weak coupling to the tidal field [13-15]. With this justification, we shall assume that the tidal field does not resonantly excite the star's normal modes.

The rotating tidal field will, however, raise a nonresonant, rotating quadrupolar tide on the neutron star. If the star's material had zero viscosity, the tide would be perfectly aligned with the tidal field; i.e., the axes of the star's rotating quadrupole moment $\mathcal{I}_{j k}$ would be identical to those of the tidal field $\mathcal{E}_{j k}$. This standard Newtonian result must be true also in general relativity since the relevant physics is nothing but that of simple harmonic oscillators: Whenever an undamped oscillator is driven by an off-resonance sinusoidal force, the oscillator's displacement response is precisely in phase with the force. As for an oscillator, so also for the star, any small viscosity will cause a slight phase lag between excitation and response: the orientation of the star's tidal deformation and its quadrupole moment will lag that of the tidal field by an angle

$$
\phi \simeq \frac{(\Omega-\omega)}{(\sigma-\omega)^{2} \tau_{*}},
$$

where $\tau_{*}$ is the viscous damping time for the star's quadrupolar excitations. For realistic viscosities, this is an exceedingly small lag angle [13-15]; so the quadrupole moment is very nearly oriented along the tidal field.

This agreement of orientations prevents the tidal field from torquing the star; so the star's spin angular momentum $J$ is conserved.

This conservation of $J$ and alignment of tidal field and quadrupole moment enable us to carry over the static-star analysis of tidal stabilization (Sec. II B) to the rotating case, essentially without change. The tidal field and quadrupole moment can be characterized by scalars in the manner of Eq. (6); raising the tide requires an energy $\delta M(Q, R)$ given by Eq. (8); the tide extracts from the tidal field an amount of energy $W(Q, \mathcal{E})$ computable by integrating the LandauLifshitz pseudotensor over a closed 2-surface in the star's 
local asymptotic rest frame in the manner of Eq. (9), and that integration must give expression (14) aside from a multiplicative factor that is close to unity. This multiplicative factor (call it $1+\gamma$ ), which we have not computed, will arise from the synchronous rotation of the tide and star with velocity $\omega r$ at the star's surface. It cannot differ from unity by more than $\gamma \sim \omega^{2} R^{2} \leq 0.01$ (there is no linear term $\gamma \sim \omega R$ because such a term would reverse sign when the star reverses direction of rotation.) As in the static case, the quadrupole moment adjusts itself so as to minimize the potential energy function $V(N, R, \mathcal{Q}, \mathcal{E})$ of Eq. (7); the resulting $Q$ is expression (15) aside from the multiplicative factor $1+\mathrm{O}\left(\omega^{2} r^{2}\right)$, and the star's radius changes slowly and secularly in a manner governed by the potential energy function $v(n, r, \varepsilon)=a r^{3}+\left(b_{0}\right.$ $\left.+b_{1} r\right) n-\left(c_{0}+c_{1} r\right) \varepsilon^{2}$ [Eq. (18)]. The only difference is in the numerical values of the coefficients $a, b_{0}, b_{1}, c_{0}$, and $c_{1}$.

As in the static case, the key to tidal stabilization is the sign of $c_{1}$, and as there, as long as the star's deformation energy at fixed quadrupole moment, $\delta M(Q, R) / Q^{2}$ $=\left(\beta / R^{5}\right) \times\left[1+\mathrm{O}\left(\omega^{2} R^{2}\right)\right]$, is a decreasing function of radius $R$, the tidal field secularly stabilizes the star, increases its radius, and decreases its central density. It is exceedingly difficult to imagine a star for which (when $\omega \leqq 2 \pi \times 600 / \mathrm{s}$ as it must be in an inspiraling binary [22]) this would not be the case.

\section{CONCLUSIONS}

In this paper we have proved that tidal fields tend to stabilize a star against secular gravitational collapse. However, for spinning stars in binaries, secular stability is irrelevant because the radiation reaction drives the binary through the regime of relativistic gravity far too quickly for viscosity to keep the rotation rigid in pulsational motions. The relevant issue in this case is dynamical stability.

As is well known (e.g., Ref. [23]), secular stability implies dynamical stability. This is so for two reasons. First, pycnonuclear reactions go to completion in secular motions but not in dynamical motions, and as a result the secular motions are characterized by a softer equation of state than the dynamical motions and thus are less stable. Second, dynamical motions of an initially rigidly rotating star produce differential rotation, and the viscous coupling that converts that differential rotation into the rigid rotation of a secular motion will necessarily extract energy from the rotation. This means that, beginning with the same equilibrium configuration, the dynamical motion must lead to a configuration of greater potential energy $V$ than the secular motion, which in turn means that the equilibrium configuration is more stable against the dynamical motion than against the secular motion.

Consider a rigidly rotating neutron star in the final, relativistic phase of binary inspiral. Such a star must have lived for millions of years to reach this inspiral phase; so it must be secularly stable as well as dynamically stable before the tidal field of its companion begins to affect it significantly. The growing tidal field, as we have seen, must increase the star's secular stability. Since secular stability implies dy- namical stability, the tidally deformed star must also remain dynamically stable.

This conclusion is not at all obvious from the equations of the post-Newtonian approximation as they are usually written: in a reference frame that is asymptotically inertial at infinity. One can identify in those equations terms that appear able to produce tidal instability at first post-Newtonian order [1]. This fact illustrates the superficially misleading character of the post-Newtonian equations: Apparently magical cancellations [3] enforce the strong equivalence principle, which is fundamentally at the heart of tidal stabilization.

By contrast, the mathematical tools used in this paper, being based on the local asymptotic rest frame of the star whose stability interests us, are closely linked to the strong equivalence principle and lead rather directly to the tidal stabilization. These tools are not widely used in relativistic astrophysics. They are worth trying whenever one is interested in the behavior of a semi-isolated portion of a larger relativistic system-e.g., a neutron star or black hole interacting with other bodies [10].

As this paper was being completed, I learned of a similar, local-asymptotic-rest-frame analysis by Flanagan [24], which, however, focuses on equations of motion rather than energy considerations.

\section{ACKNOWLEDGMENTS}

For helpful discussions and/or comments on the manuscript, the author thanks Roger Blandford, Patrick Brady, Doug Eardley, Eanna Flanagan, Scott Hughes, Dong Lai, Patricia Purdue, Stuart Shapiro, and Alan Wiseman. This research was supported in part by NSF Grant AST-9417371.

\section{APPENDIX}

In this appendix, we elucidate the energetics of quadrupole-tidal coupling, Eqs. (9)-(14), by working out its details in the Newtonian limit without rotation.

We consider a nonroting Newtonian star that is spherical, aside from a quadrupolar deformation and quadrupole moment $\mathcal{I}_{j k}$ that are aligned with an external tidal field $\mathcal{E}_{j k}$ $=\Phi_{\mathrm{e}, j k}$. Here $\Phi_{\mathrm{e}}$ is the external Newtonian potential, and $\mathcal{E}_{j k}$ and $\mathcal{I}_{j k}$ are symmetric and trace free. We denote by $\rho, p$, $v$ and $\Pi$ the (Newtonian) mass density, pressure, velocity and specific internal energy of the stellar fluid, and by

$$
\Phi=\Phi_{\mathrm{o}}+\Phi_{\mathrm{e}}
$$

the Newtonian gravitational field and its split into the star's self-field $\Phi_{\mathrm{o}}$ and the external field $\Phi_{\mathrm{e}}$.

In Newtonian theory, the total energy density and energy flux of the stellar fluid plus gravitational field can be written as

$$
\begin{aligned}
& \Theta^{00}=\rho\left(\frac{1}{2} v^{2}+\Pi+\Phi\right)+\frac{1}{8 \pi} \Phi_{, j} \Phi_{, j}, \\
& \Theta^{0 j}=\rho v^{j}\left(\frac{1}{2} v^{2}+\Pi+\frac{p}{\rho}+\Phi\right)-\frac{1}{4 \pi} \Phi_{, t} \Phi_{, j},
\end{aligned}
$$


where we make no distinction between covariant and contravariant spatial indices because our coordinates are assumed to be Cartesian. It is straightforward to verify that the energy conservation law

$$
\Theta^{00}{ }_{, t}+\Theta^{0 j}{ }_{j}=0
$$

is satisfied by virtue of mass conservation $\rho_{, t}+\left(\rho v^{j}\right)_{, j}=0$, the first law of thermodynamics, $\rho d \Pi / d t+p v^{j}{ }_{j}=0$, the fluid's Euler equation $\rho d v^{j} / d t+\rho \Phi_{, j}+p_{, j}=0$, and Newton's field equation $\Phi_{, j j}=4 \pi \rho$. Here $d / d t=\partial / \partial t+v^{j} \partial / \partial x^{j}$ is the fluid's comoving time derivative.

[Note that Eqs. (A2) and (A3) entail a specific choice of how to localize the system's gravitational energy. Other choices are possible: one can add to $\Theta^{00}$ the divergence of $\eta_{j} \equiv \alpha \Phi \Phi_{, j}$ (where $\alpha$ is an arbitrary constant) and add to $\Theta^{0 j}$ minus the time derivative of $\eta_{j}$ without affecting the law of energy conservation (A4) or any of the system's physics. This nonuniqueness of localization of gravitational energy also occurs in general relativity; cf. Chap. 20 of MTW [11]. We shall discuss its consequences below.]

The external field is purely quadrupolar and source free throughout the star and the star's local asymptotic rest frame

$$
\Phi_{\mathrm{e}}=\frac{1}{2} \mathcal{E}_{i j} x^{i} x^{j}, \quad \Phi_{\mathrm{e}, j j}=0,
$$

and its tidal field $\mathcal{E}_{i j}$ evolves with time. The star's external self-field is purely monopolar and quadrupolar and its source, of course, is the star's mass distribution

$\Phi_{\mathrm{o}}=-\frac{M}{r}-\frac{3}{2} \frac{\mathcal{I}_{i j} n^{i} n^{j}}{r^{3}}$ outside star, $\Phi_{\mathrm{o}, j j}=4 \pi \rho$.

Here $r \equiv \sqrt{\delta_{i j} x^{i} x^{j}}$ is the radius and $n^{j} \equiv x^{j} / r$ is the unit radial vector. The star's mass $M$ is constant in time, but its quadrupole moment $\mathcal{I}_{i j}$ evolves.

Now consider the total energy $E$ inside a ball $\mathcal{V}$ centered on the star and larger than the star. This energy consists of three parts, the star's self-energy $E_{\mathrm{o}}$, the external field's energy $E_{\mathrm{e}}$, and the energy $E_{\text {int }}$ of interaction between the star's quadrupolar deformation and the external tidal field:

$$
\begin{gathered}
E \equiv \int_{\mathcal{V}} \Theta^{00} d^{3} x=E_{\mathrm{o}}+E_{\mathrm{e}}+E_{\mathrm{int}}, \\
E_{\mathrm{o}}=\int_{\mathcal{V}}\left[\rho\left(\frac{1}{2} v^{2}+\Pi+\Phi_{\mathrm{o}}\right)+\frac{1}{8 \pi} \Phi_{\mathrm{o}, j} \Phi_{\mathrm{o}, j}\right] d^{3} x, \\
E_{\mathrm{e}}=\int_{\mathcal{V}} \frac{1}{8 \pi} \Phi_{\mathrm{e}, j} \Phi_{\mathrm{e}, j} d^{3} x, \\
E_{\mathrm{int}}=\int_{\mathcal{V}}\left(\rho \Phi_{\mathrm{e}}+\frac{1}{4 \pi} \Phi_{\mathrm{o}, j} \Phi_{\mathrm{e}, j}\right) d^{3} x=\frac{3}{10} \mathcal{E}_{i j} \mathcal{I}_{i j} .
\end{gathered}
$$

Here the value of $E_{\text {int }}$ follows from the form and sourcelessness (A5) of the external field, the source equation and ex- terior form of the star's self-field (A6), and the usual volume integral for the quadrupole moment $\mathcal{I}_{i j}=\int_{\mathcal{V}} \rho\left(x^{i} x^{j}\right.$ $\left.-\frac{1}{3} r^{2} \delta^{i j}\right) d^{3} x$.

The law of local energy conservation (A4) guarantees that the rate of change of the total energy (A7) is the surface integral of the energy flux over the boundary $\partial \mathcal{V}$ of the ball $\mathcal{V}:$

$$
\frac{d E}{d t}=-\int_{\partial \mathcal{V}} \Theta^{0 j} n^{j} r^{2} d \Omega
$$

Here $d \Omega$ is solid angle. By combining Eqs. (A11), (A3), (A1), (A5), (A6) and (A9), we find that

$$
\begin{aligned}
\frac{d E}{d t}= & \frac{d E_{\mathrm{e}}}{d t}+\int_{\partial \mathcal{V}} \frac{1}{4 \pi} \Phi_{\mathrm{o}, t} \Phi_{\mathrm{o}, j} n^{j} r^{2} d \Omega \\
& +\frac{d}{d t}\left(\frac{3}{10} \mathcal{I}_{j k} \mathcal{E}_{j k}\right)-\frac{1}{2} \mathcal{E}_{j k} \frac{d}{d t} \mathcal{I}_{j k} .
\end{aligned}
$$

The first term is the rate of change of the external field energy, inside the ball, due to the evolution of the tidal field $\mathcal{E}_{j k}$. The second term is the rate of change of the star's self-energy due its own field energy flowing into or out of the ball $\mathcal{V}$ as its quadrupole moment decreases or increases; in the limit as the ball's radius becomes arbitrarily large, this term goes to zero. The third term is the rate of change of the interaction energy; cf. Eq. (A10). By comparing with Eq. (A7), we see that the last term must drive changes in the star's self-energy:

$$
\frac{d E_{\mathrm{o}}}{d t}=\int_{\partial \mathcal{V}} \frac{1}{4 \pi} \Phi_{\mathrm{o}, t} \Phi_{\mathrm{o}, j} n^{j} r^{2} d \Omega-\frac{1}{2} \mathcal{E}_{i j} \frac{d}{d t} \mathcal{I}_{i j} .
$$

In other words, the last term is the rate at which the external tidal field does work on the star:

$$
\frac{d W(\mathcal{Q}, \mathcal{E})}{d t}=-\frac{1}{2} \mathcal{E}_{i j} \frac{d}{d t} \mathcal{I}^{i j} .
$$

This work rate is in perfect agreement with the fully relativistic work rate (12) derived in the text using the LandauLifshitz pseudotensor to localize the gravitational energy and using deDonder (harmonic) gauge.

This is not so for the rate of change of the interaction energy. The Newtonian analysis gives

$$
\left(\frac{d E_{\text {int }}}{d t}\right)_{\text {Newton }}=\frac{3}{10} \frac{d\left(\mathcal{E}_{i j} \mathcal{I}^{i j}\right)}{d t}
$$

[Eq. (A10) above]; by contrast, the relativistic analysis gives this same expression but with a coefficient $-1 / 10$ rather than $+3 / 10$; see Eq. (13). This disagreement does not arise from any fundamental difference between Newtonian theory and general relativity. Rather, it arises because the localization of gravitational energy is non-unique, both in Newtonian theory and in general relativity [see the paragraph preceding Eq. (A5)], and $E_{\text {int }}$ is that portion of the system's total gravitational interaction energy which resides in the vicinity of the 
particular neutron star on which we are focusing. For a binary system, our two analyses distribute the gravitational energy differently between the stars' vicinities and the interstellar region, and they thereby give rise to different values of $E_{\text {int }}$. If, in the Newtonian analysis, we were to change our localization of the gravitational energy via the transformation described in the paragraph preceding Eq. (A5), we would alter $E_{\text {int }}$, while leaving unchanged the uniquely defined work $W$ done by the tidal field on the star of interest. Similarly, if, in our general relativitistic analysis, we were to change our energy localization by switching from the Landau-Lifshitz pseudotensor to some other pseudotensor, or by performing a gauge change on the gravitational field, we thereby would alter $E_{\text {int }}$ but leave $W$ unchanged.

This localization dependence of $E_{\text {int }}$ and uniqueness of $W$ will be analyzed in detail in a subsequent paper [18].
[1] J. R. Wilson and G. J. Mathews, Phys. Rev. Lett. 75, 4161 (1995); J. R. Wilson, G. J. Mathews, and P. Maronetti, Phys. Rev. D 54, 1317 (1996).

[2] D. Lai, Phys. Rev. Lett. 76, 4878 (1996); see also J. C. Lombardi, F. A. Rasio, and S. L. Shapiro, Phys. Rev. D 56, 3416 (1997).

[3] A. D. Wiseman, Phys. Rev. Lett. 79, 1189 (1997).

[4] P. R. Brady and S. A. Hughes, Phys. Rev. Lett. 79, 1186 (1997).

[5] T. W. Baumgarte, G. B. Cook, M. A. Scheel, S. L. Shapiro, and S. A. Teukolsky, Phys. Rev. Lett. 79, 1182 (1997); Phys. Rev. D 57, 6181 (1998).

[6] B. K. Harrison, K. S. Thorne, M. Wakano, and J. A. Wheeler, Gravitation Theory and Gravitational Collapse (University of Chicago Press, Chicago, 1965).

[7] S. L. Shapiro and S. A. Teukolsky, Black Holes, White Dwarfs, and Neutron Stars (Wiley, New York, 1983).

[8] D. W. Meltzer and K. S. Thorne, Astrophys. J. 145, 514 (1966), especially Table 1 and Figs. 1 and 4.

[9] K. S. Thorne, Rev. Mod. Phys. 52, 299 (1980).

[10] K. S. Thorne and J. B. Hartle, Phys. Rev. D 31, 1815 (1985).

[11] C. W. Misner, K. S. Thorne, and J. A. Wheeler, Gravitation (Freeman, San Francisco, 1973).
[12] Y. Gürsel, Gen. Relativ. Gravit. 15, 737 (1983), Sec. B of the Appendix.

[13] D. Lai, Mon. Not. R. Astron. Soc. 270, 611 (1994).

[14] A. Reisenegger and P. Goldreich, Astrophys. J. 426, 688 (1994).

[15] M. Shibata, Prog. Theor. Phys. 91, 871 (1994).

[16] W. Thomson (Lord Kelvin), Philos. Trans. R. Soc. London 153, 612 (1863); also published in Sir William Thomson, Mathematical and Physical Papers (C. J. Clay and Sons, Cambridge University Press Warehouse, London, 1890), Vol. 3, p. 384.

[17] L. D. Landau and E. M. Lifshitz, The Classical Theory of Fields (Pergamon, Oxford, 1975).

[18] P. M. Purdue and K. S. Thorne (in preparation).

[19] X.-H. Zhang, Phys. Rev. D 31, 3130 (1985).

[20] X.-H. Zhang, Phys. Rev. D 34, 991 (1986).

[21] J. B. Hartle and K. S. Thorne, Astrophys. J. 153, 807 (1968).

[22] D. Lai and A. G. Wiseman, Phys. Rev. D 54, 3958 (1996).

[23] J. L. Friedman, J. R. Ipser, and R. D. Sorkin, Astrophys. J. 325, 722 (1988).

[24] E. E. Flanagan, preceding paper, Phys. Rev. D 58, 124030 (1998). 